\title{
Multidetector Computer Tomography in Evaluation of Ovarian Vein Reflux
}

\author{
Authors \\ Mohamed A A, Mamdouh H H, Chepsiror D K \\ Radiology Department, Faculty of Medicine, Alexandria University
}

\begin{abstract}
Objective: The aim of this study was to determine the role of MDCT in evaluating ovarian vein reflux, identify possible cause and grade its degree.

Materials And Methods: Contrast enhanced MDCT images of five hundred and nine (509) female patients scanned between $1^{\text {st }}$ of March 2015 and $31^{\text {st }}$ of August 2015 were reviewed for the evaluation of ovarian veins (normal or pathological states) and any associated abdominall pelvic pathology/pathologies. The presence, degree, pathway and associated findings of ovarian vein reflux were evaluated.

Results: From the 509 patients, $1006 / 1018$ (98.82\%) ovarian veins were identified. There were fifty six $(56 / 509,11 \%)$ female patients who became study subjects with ovarian vein reflux distributed as follows. A total of sixty eight out of 1006 (6.76\%) identified ovarian veins had reflux. Right only were 12 OVs, left only, $32 \mathrm{OVs}$ and (12 cases) 24 OVs were bilateral. Majority, 51/68 (75\%) of refluxing OVs were of secondary type (identifiable cause) and 17/68 (25\%) were of primary type (no identifiable cause). Most of the refluxing OVs $(38 / 68,55.88 \%)$ were of grade II, followed grade I (20/68, 29.41\%) while those of grade III were $10(14.71 \%)$. Grade III had statistical significance in its occurrence on the left OVs compared to the right OVs ( $p=0.011)$.

Conclusion: Both primary and secondary $O V$ reflux exist. Left ovarian vein was the most affected by reflux as well as the severe grade III seen extending to contralateral ovarian vein plexus by passing through the dilated para-uterine and uterine veins with none affecting the right one. In overall, grade II reflux was common on both OVs.
\end{abstract}

\section{Introduction}

The aim of this study was to determine the role of MDCT in evaluating ovarian vein reflux, identify possible cause and grade its degree. During the study period a total of five hundred and nine patients were included in the study from female patients who were referred to radiology department. One thousand and six (1006) out of the expected 1018 OVs were identified.

Pelvic venous insufficiency and varicosities, one of commonly missed and potentially treatable cause of chronic abdominal or pelvic pain is a condition that results from retrograde flow through incompetent valves in ovarian veins either congenital or acquired.

The causes of ovarian vein varicosities are multifactorial and studies have described multiple factors including hereditary factors, hormonal influence, pelvic surgery, retroverted uterus, a history of varicose veins, and multiple pregnancies. Associated findings of cystic ovaries are found in over $50 \%$ of cases. ${ }^{(1-4)}$

The primary defect is the absence of functioning valves, resulting in retrograde blood flow and 
eventually venous dilatation. Valves are absent from the orifices of the gonadal veins in $15 \%$ of women, and, in those where valves are present, they are incompetent in $40 \%$ on the left and $35 \%$ on the right. ${ }^{(5,6)}$

The condition is more common in multiparous, premenopausal women who typically present with chronic pelvic pain for more than 6 months with overall population prevalence approaching $\sim 10 \%$. Within this group of patients, up to $60 \%$ may develop pelvic congestion syndrome. In general, pelvic congestion syndrome is considered an underdiagnosed cause of chronic pelvic pain because of the non-specificity of the observations made with conventional imaging. However, with recent advances in imaging technology, a substantial number of women can benefit from an accurate diagnosis. ${ }^{(7,8)}$

CT, MRI, and selective ovarian venography show incompetent dilated ovarian veins and pelvic varices. Some authors have reported that reflux in the left ovarian vein leads to the development of pelvic varices. $^{(3)}$

Ovarian vein reflux is an interesting phenomenon because it can be seen to some degree in diseasefree women, but it can also be a sign of disease (e.g., pelvic congestion syndrome [PCS]). PCS is characterized by chronic pelvic pain of at least 6 months' duration without any identifiable organic cause. $^{(2,3)}$ and most patients with PCS are multiparous women.

\section{Materials and Methods \\ Patients}

MDCT images of five hundred and nine (509) female patients scanned between $1^{\text {st }}$ of March 2015 and $31^{\text {st }}$ of August 2015 were reviewed for evaluation of ovarian veins (normal or pathological states) and any associated abdominal or pelvic pathology/pathologies.

The purpose of this study was to assess ovarian veins in all female patients referred to Radiology department for contrast enhanced MDCT.
Excluded patients included those who were pregnant and those with absolute contraindication to intravenous contrast administration.

For the study subject selection, we took a thorough medical history, did a complete physical examination, careful review of medical records and previous/current imaging studies of all patients.

Preliminary review of current MDCT images was done, those cases with refluxing ovarian veins were selected and further interpretation done. There werefifty six $(56 / 509,11 \%)$ female patients who became study subjects with ovarian vein reflux distributed as follows. A total of sixty eight ovarian veins with reflux were detected. Twelve on the right side only, thirty two affected the left side only and bilateral in twelve cases (twenty four ovarian veins).

\section{MDCT Technique}

A non-contrast scan was done followed by intravenous contrast administration in all patients forming the study group. Nonionic Optiray (Ioversol 64\%, tyco Healthcare, Mallinckrodt, USA) $300 \mathrm{mg}$ Iodine/ml intravenous contrast material was used. Adult dose was $1-2 \mathrm{mls} / \mathrm{kg}$ (maximum $150 \mathrm{mls}$ ) while pediatric dose was 1$1.5 \mathrm{ml} / \mathrm{kg}$. Administration was done at a flow rate of $2-5 \mathrm{ml} / \mathrm{s}$ depending on the indication of the examination and condition of the patient. Scanning was initiated after a 18-25 sec for arterial phase, followed by repeated scanning with 60-70 s delay for obtaining the venous phase (at which the ovarian veins are highlighted and is considered the best timing for their evaluation), this was followed by the delayed venous equilibrium phase,(important for demonstration of the collecting system of the kidneys and the urinary bladder cavity opacification).

\section{Scan parameters}

The examinations were done using Siemens 64 slices (Somatom, Germany), Philips 16 slices and Philips 64 slices (Philips healthcare. Best, Netherlands) MDCT scanners.

Scanning parameters were: $125 \mathrm{mAs}, 130 \mathrm{KVP}$, tube rotation time of $1.19 \mathrm{~s} / \mathrm{rot}, 1.5 \mathrm{~mm}$ slice 
thickness and $1.5 \mathrm{~mm}$ reconstruction interval for Siemens 64 slices MDCT, while for Philips 16 slices MDCT were; $200 \mathrm{mAs}, 140 \mathrm{KVP}$, tube rotation time of $1.0 \mathrm{~s} / \mathrm{rot}, 2.0 \mathrm{~mm}$ slice thickness and $1.5 \mathrm{~mm}$ reconstruction interval and $30 \mathrm{mAs}$, $140 \mathrm{kvp}$, tube rotation time of $1.25 \mathrm{~s}$ and slice thickness of $2.0 \mathrm{~mm}$ for Philips 64 slices MDCT.

\section{Reconstruction methods}

The volume of data formed by the thin slice images was sent to IntelliSpace Portal Philips workstation and Osirix workstation for Philips and Siemens MDCT scanner machines respectively, where they were available to view the data in axial, sagittal and coronal planes. Thin slice acquisition and multi-planar capability allowed accurate identification and tracking of both ovarian veins on all patients on both sides when found. This was done from their origin at the pelvic region to termination and determination of their normal or pathological states as well other abdomino-pelvic disorders. Advanced post processing techniques including maximum intensity projection (MIP) images were applied for better demonstration of the contrast enhanced vascular lumen along their whole course.

\section{MDCT evaluation}

Retrospective analysis of reconstructed MDCT scans was performed independently by radiologists who reviewed the scans looking for
OVs and for presence of any abdominal/pelvic pathology. This enabled classification of $\mathrm{OV}$ reflux into either primary (no identifiable cause) or secondary, where the cause of reflux was identified.

\section{Interpretation}

The ovarian veins were next evaluated for caliber and incompetence (reflux). An ovarian vein was considered as dilated when it measured $4 \mathrm{~mm}$ or greater in its maximum diameter. Veins smaller than $1 \mathrm{~mm}$ were categorized as attenuated while normal were between 1-4mm.

An ovarian vein was considered incompetent if it was completely opacified with IV contrast material during the arterial/ corticomedullary kidney phase of CE-MDCT. Medical records of all subjects were reviewed with attention paid to any abdominal pain or gynecologic complaints. Applying the classification by Tadao (2004), ${ }^{(1)}$ the flow-pathway was assessed from the level of the kidneys to the pelvic floor. From this, the degree of reflux was divided into three grades. In grade I, retrograde flow remained in the ovarian vein, not reaching the para-uterine veins. In grade II, the retrograde flow extended to the ipsilateral parauterine veins and not farther. Finally, in grade III, the retrograde flow crossed the midline passing through the uterus from the left into the right parauterine plexus and vice versa.

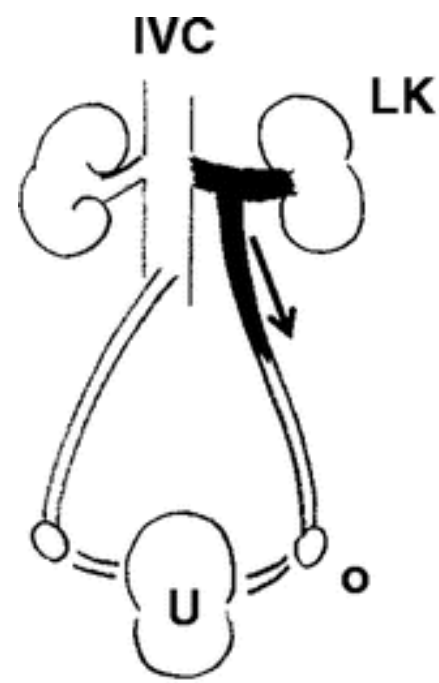

Grade I

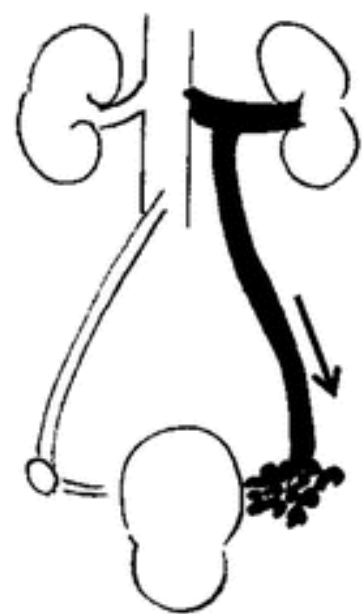

Grade II

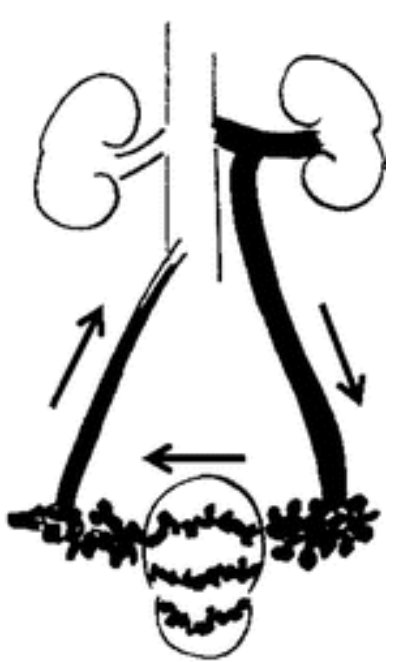

Grade III

Drawing of anterior view of ovarian veins shows degrees of ovarian vein reflux. IVC = inferior vena cava, LK = left kidney, $\mathrm{U}=$ uterus, $\mathrm{o}=$ ovary 


\section{Results}

Distribution of 68 refluxing veins according to the cause and side affected

A total of sixty eight out of 1006 identified ovarian veins had reflux. Right only were $12 \mathrm{OVs}$, left only, 32 OVs and (12 cases) 24 OVs were bilateral. Majority, 51 (75\%) OVs were secondary to other abdominal pathologies and 17 (25\%) OVs were primary (no identifiable cause) (table I)

Table I: Distribution of 68 dilated refluxing veins according to the side affected

\begin{tabular}{|l|l|l|l|l|l|}
\hline & Right OV & Left OV & Bilateral OV & Total OV & $\%$ \\
\hline $\begin{array}{l}\text { Reflux- } \\
\text { Primary }\end{array}$ & 4 & 11 & 2 & 17 & 25 \\
\hline -Secondary & 8 & 23 & 10 & 51 & 75 \\
\hline Total & 12 & 32 & 24 & 68 & \\
\hline
\end{tabular}

Correlation between reflux with patients' age and side affected

The mean age of patients with refluxing OVs was 46.7 years (range 25-75 years). It occurred mostly in patients in $6^{\text {th }}$ decade $(25,36.76 \%)$ followed by those in $5^{\text {th }}$ decade $(17,25 \%)$ and the least in $7^{\text {th }}$ and $8^{\text {th }}$ decades (four each, $5.88 \%$ ). No refluxing veins were detected in $1^{\text {st }}, 2^{\text {nd }}$ or $9^{\text {th }}$ decade.

Correlation between main abdomino-pelvic pathology with ovarian vein reflux and side affected

Most of the refluxing ovarian veins, thirteen $(19.11 \%)$ were found in patients with hepatocellular carcinoma with/without compression of intra-hepatic segment of IVC, with or without cirrhosis and some also had portal hypertension. They were followed by patients with inflammatory disorderswho presented for evaluation with imaging $(10,14.71 \%)$, then congenital vascular malformation which were nine, (13.24\%) ( four in two patients with absent infra-renal vena cava, one in a patient with double IVC and four in patients who had retro-aortic course of left renal vein). The least incidence occurred in patients with abdominal mass, cancer of the stomach and ovary, trauma and hepatic adenoma, one each, $(1.47 \%)$.

Correlation between ovarian vein caliber and reflux

All the refluxing ovarian veins were dilated, Twenty five veins were found in $8-<10 \mathrm{~mm}$ category, followed by 23 in $6-<8 \mathrm{~mm}$ and least were those with calibers $4-<6 \mathrm{~mm}$.

Compared with dilatation, most refluxing OVs, $(10 / 14,71.42 \%)$ occurred at $10-<12 \mathrm{~mm}$ calibers, followed by $(6 / 9,66.67 \%)$ in those with $>12 \mathrm{~mm}$ caliber while least $(4 / 70,5.71 \%)$ were in $4-<6 \mathrm{~mm}$ category. Reflux in OVs were statistically significant in $8-<10 \mathrm{~mm}$ and $10-<12 \mathrm{~mm}$ (p-0.004 and .0027 respectively) (Table II)

Table II: Correlation between ovarian vein caliber and reflux

\begin{tabular}{|l|l|l|l|l|l|l|}
\hline & Dilated & \multicolumn{3}{|c|}{ Reflux } & p & $\begin{array}{l}\text { \% reflux of } \\
\text { dilated veins }\end{array}$ \\
\hline $\begin{array}{l}\text { Caliber } \\
(\mathrm{mm})\end{array}$ & & Right & Left & Total & & \\
\hline $4-<6$ & 70 & 1 & & & & \\
\hline $6-<8$ & 86 & 9 & 3 & 4 & $<0.001^{*}$ & 5.71 \\
\hline $8-<10$ & 44 & 8 & 14 & 23 & 0.479 & 26.74 \\
\hline $10-<12$ & 14 & 4 & 17 & 25 & $0.004^{*}$ & 56.81 \\
\hline$>12$ & 9 & 2 & 6 & 10 & $0.027^{*}$ & 71.42 \\
\hline & 223 & 24 & 4 & 6 & 0.125 & 66.67 \\
\hline
\end{tabular}

$\mathrm{p}$ : $\mathrm{p}$ for Chi square test

*: Statistically significant at $\mathrm{p} \leq 0.05$ 
Grading of ovarian vein reflux

After assessing the flow-pathway from the level of the kidneys to the pelvic floor, the degree of reflux was divided into three grades. In grade I, retrograde flow remained in the left or right ovarian vein, not reaching the para-uterine veins. (Figure 1). In grade II, the retrograde flow extended to the ipsilateral para-uterine veins and not farther. (Figure 2). Finally, in grade III, (Figure 3 and 4), the retrograde flow crossed the midline passing through the uterus from the left into the right para-uterine plexus and vice versa.
Refluxing ovarian veins were forty four on the left side while 24 were on the right side. Grading of refluxing veins showed that most of the refluxing OVs $(38 / 68,55.88 \%)$ were of grade II, followed grade I $(20 / 68,29.41 \%)$ while those of grade III were $10(14.71 \%)$. For grade III, there was a statistical significant difference between right and left $(\mathrm{p}=0.011)$

Overally, grade II reflux was common on both sides, being 10 on right and 28 on the left side. (Table III)

Table III: Grading of ovarian vein reflux

\begin{tabular}{|l|l|l|l|l|l|}
\hline Grade & Right & Left & FEp & Total & Percent \\
\hline I & 6 & 14 & 0.555 & 20 & 29.41 \\
\hline II & 10 & 28 & 0.081 & 38 & 55.88 \\
\hline III & 0 & 10 & $0.011^{*}$ & 10 & 14.71 \\
\hline Total & 24 & 44 & & 68 & \\
\hline MCp & 0.150 & & & \\
\hline
\end{tabular}

MC: Monte Carlo for Chi square test

FE: Fisher Exact for Chi square test

*: Statistically significant at $\mathrm{p} \leq 0.05$

\section{Images}

\section{Case 1}

Grade I reflux

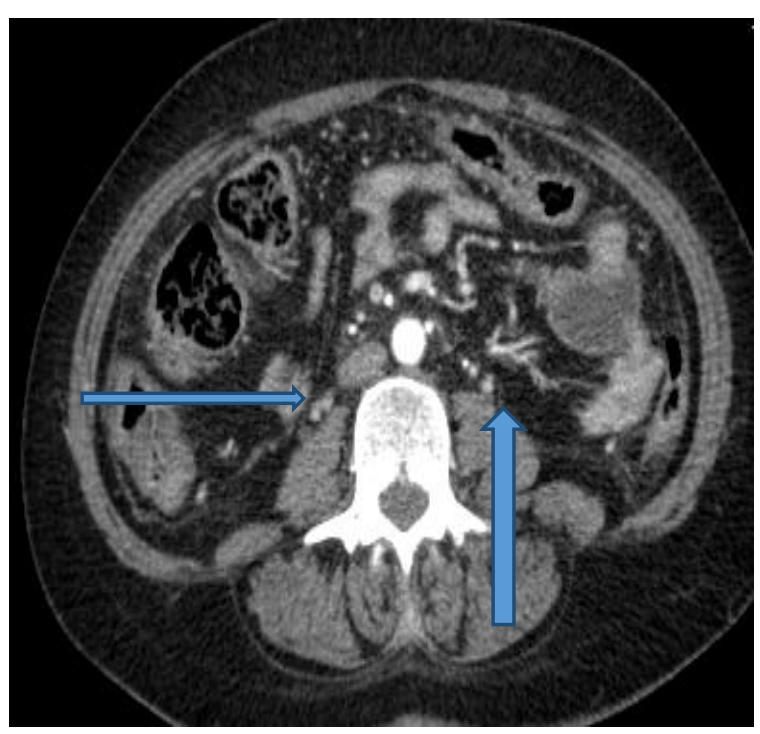

a)

Figure 1: A forty two year old patient with history of breast cancer managed by surgery and chemotherapy on follow up. Abdominal CE-CT showed mildly dilated ovarian veins. The

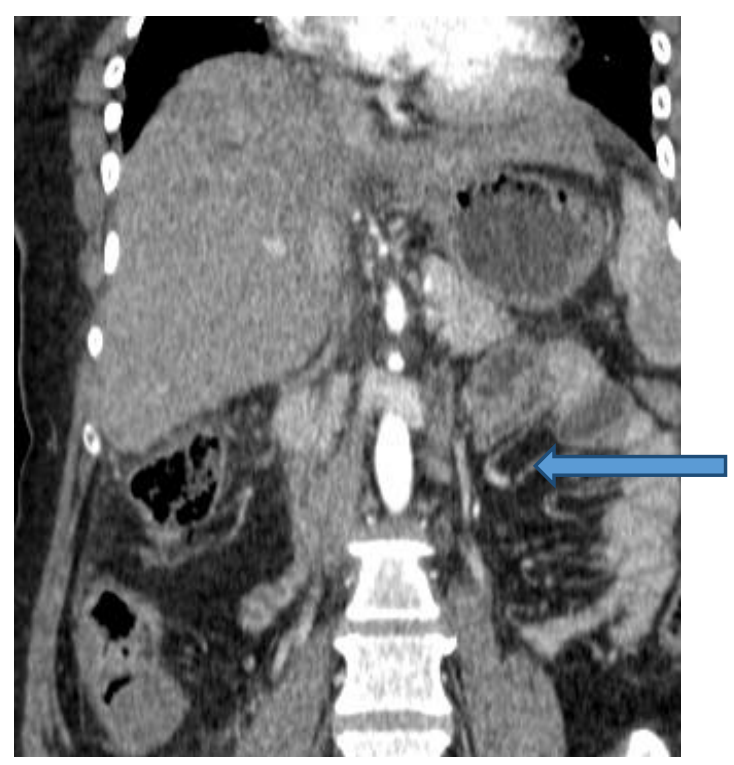

b)

proximal portions of both ovarian veins are seen filling with contrast in arterial phase of the study (indicating reflux). (blue arrows in a and b) but no extension to para-uterine veins-Grade I 


\section{JMSCR Vol||4||Issue||02||Page 9072-9082||February}

\section{Case 2}

Grade II reflux

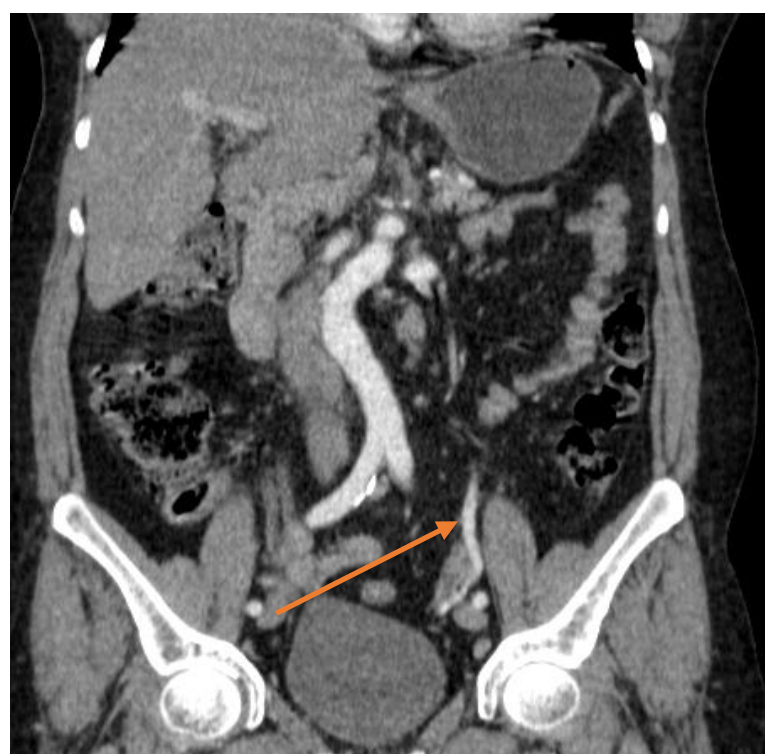

a)

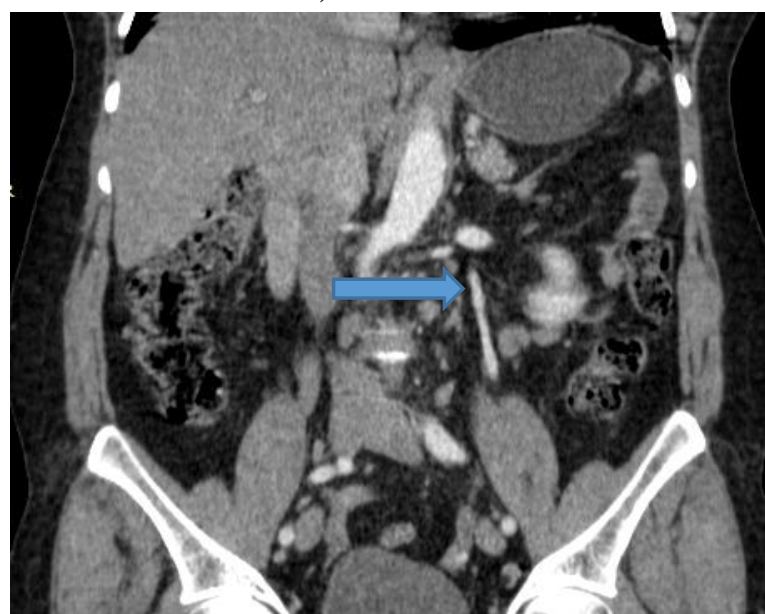

c)

Figure 2: A fifty seven year old female patient with previous history of colon cancer managed by surgery and chemotherapy. She had no complains but came for routine follow up. Abdonino-pelvic MDCT was unremarkable. However, the left ovarian vein is seen mildly dilated, measuring 5

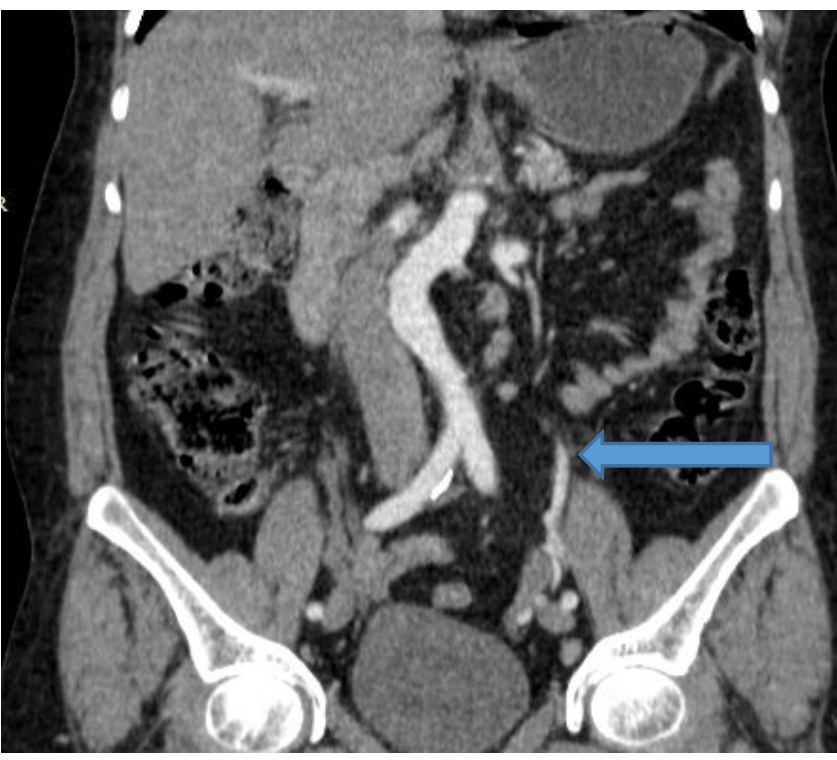

b)

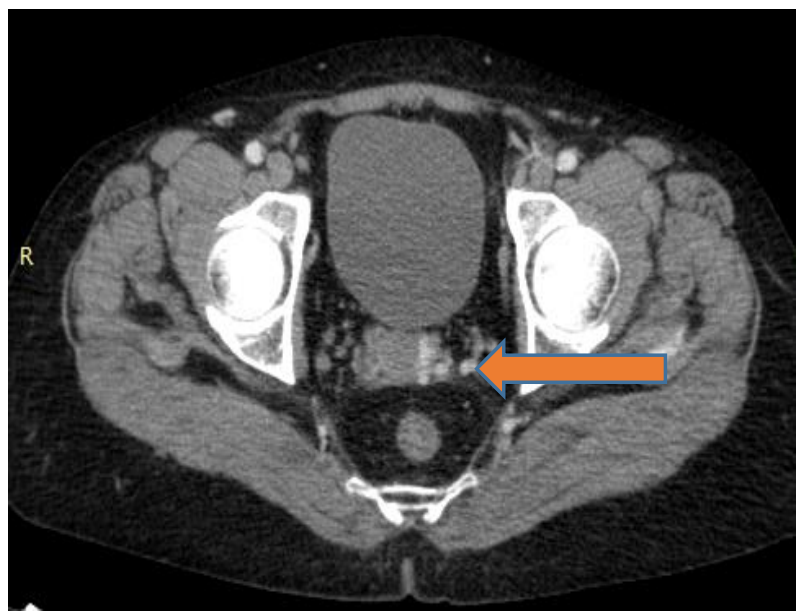

d)

$\mathrm{mm}$ in caliber and filling with contrast during arterial phase denoting presence of reflux.(arrow in a-c). Reflux extends to involve left para-uterine venous radicals and no extension to right side. (arrow in d), grade II. 


\section{JMSCR Vol||4||Issue||02||Page 9072-9082||February}

Case 3

Grade III reflux

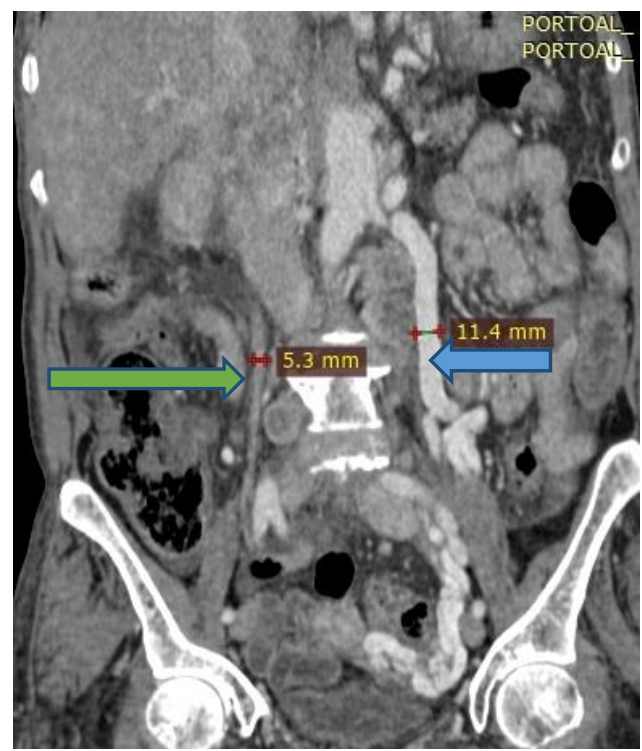

a)

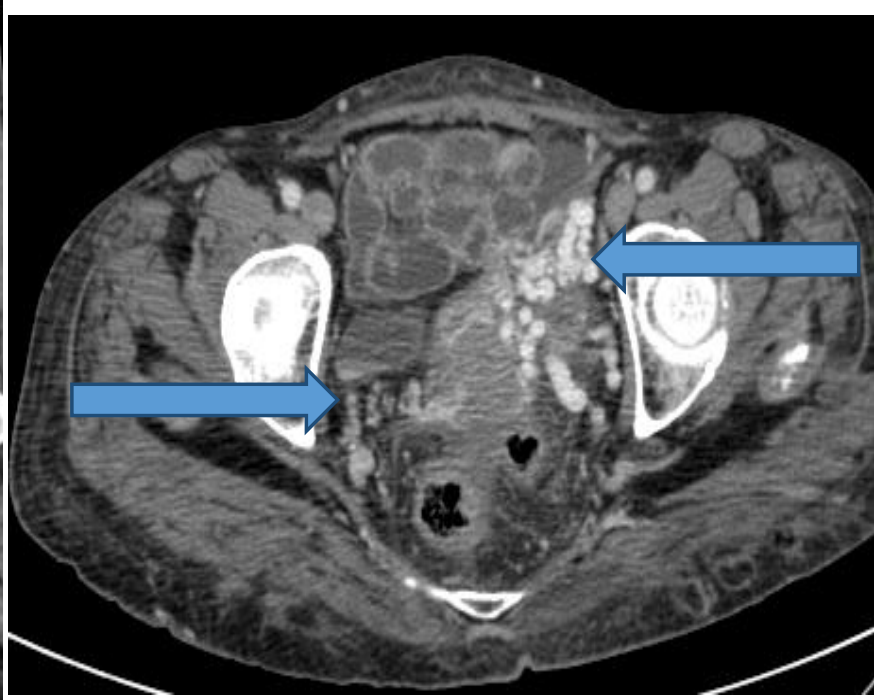

b)

Figure 3: A seventy two year old patient with known history of HCC of follow-up. Physical examination revealed a large epigastric-right hypochondrial mass with tenderness over the whole abdomen and pelvis but more on the left side. CE-CT showed a large partially exophytic hepatic mass with wash in wash out pattern of enhancement It compressed the hepatic segment of IVC with back pressure. The left ovarian vein is seen tortuous and dilated measuring 11.4 in

Case 4

Grade III reflux

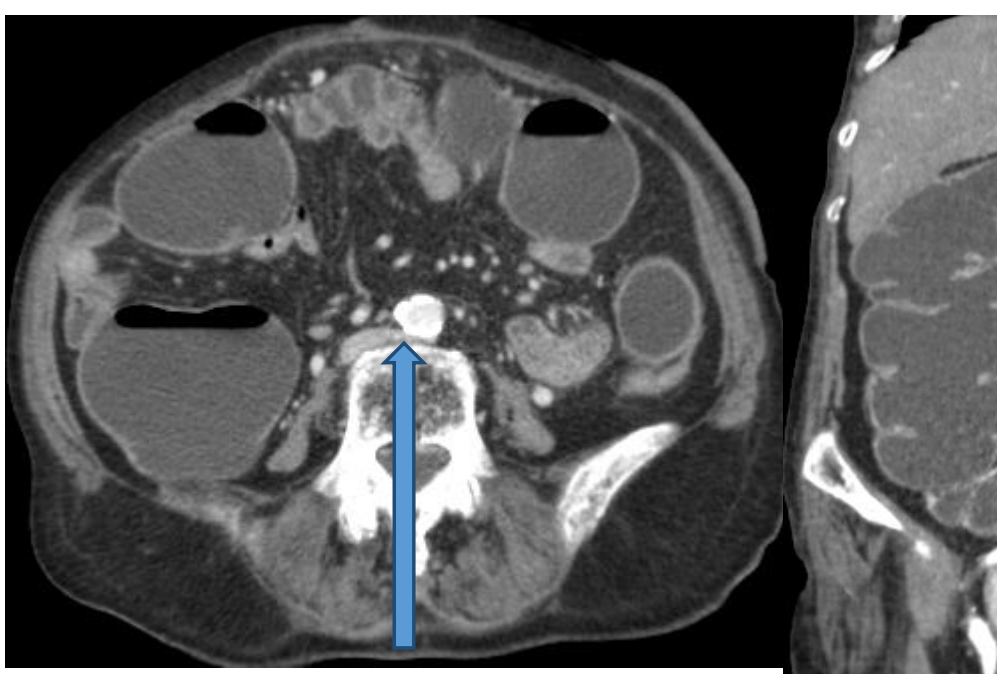

a) caliber showing full length opacification in arterial phase of the study indicating reflux (blue arrow in a). The right ovarian vein is also dilated but not refluxing (green in a). Scans through the pelvis shows multiple worm-like dilated bilateral pelvic and parauterine veins all filling with contrast arterial phase representing grade III reflux. (arrows in b). 


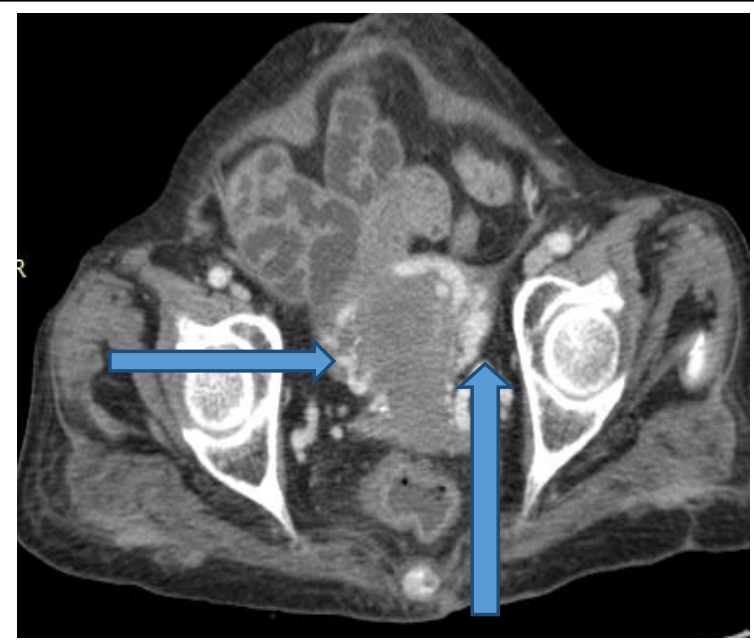

c)

Figure 4: A sixty five year old female patient with history of recto-sigmoid cancer managed by surgery came for follow up and also had on and off abdominal pain. Physical examination revealed generalized mild abdominal tenderness. CE-CT of the abdomen showed calcular cholecystitis and dilated large bowel loops. A retro-aortic left renal vein was noted compressed between the aorta and vertebral body. (Arrow in a). Left ovarian vein is dilated, measuring $8.2 \mathrm{~mm}$ in caliber and filling with csontrast during arterial phase denoting reflux. (arrow in c) It extends to involve dilated pelvic and para-uterine veins bilaterally. (arrows in c), Grade III.

\section{Discussion}

Ovarian (female gonadal) veins are paired veins that originate from a pampiniform plexus in the broad ligament near the ovary and fallopian tube which communicate with the uterine plexus. They then merge into a single vein on each side which course anterior to the psoas muscle and the ureter. The right ovarian vein drains into the inferior vena cava and the left ovarian vein into the left renal vein in most individuals ${ }^{(1-3)}$

The aim of this study was to determine the role of MDCT in evaluating ovarian vein reflux, identify possible cause and grade its degree. During the study period a total of five hundred and nine patients were included in the study from female patients.

The current work included five hundred and nine female patients who were referred to radiology department for contrast enhanced MDCT. One thousand and six (1006) out of the expected 1018 OVs were identified. Sixty eight (68/1006, 6.76\%) refluxing ovarian veins were detected distributed as follows; twelve on the right side only, thirty two affected the left side only and bilateral in twelve cases (twenty four ovarian veins).

$\mathrm{OV}$ vein reflux is an interesting phenomenon because it can be seen to some degree in diseasefree women, but it can also be a sign of disease (e.g., pelvic congestion syndrome [PCS]) and most patients with PCS are multiparous women. ${ }^{(1)}$ CT, MRI, and selective ovarian venography show incompetent dilated ovarian veins and pelvic varices. (3) Additionally,some authors have reported that reflux in the left ovarian vein leads to the development of pelvic varices. ${ }^{(9-14)}$

The causes of ovarian vein varicosities are multifactorial and studies have described multiple factors including hereditary factors, hormonal influence, pelvic surgery, retroverted uterus, a history of varicose veins, and multiple pregnancies. Associated findings of cystic ovaries are found in over $50 \%$ of cases. ${ }^{(1-4)}$

This was also replicated in the current study where patients had variable presenting complains and other abdominal pathologies while some had no predisposing factors.

Among patients with OV reflux, MDCT typically shows tortuous, dilated structures more than $4 \mathrm{~mm}$ in the uterine adnexa or besides the ureter which are iso-dense to other abdominal veins on postcontrast venous phase images. The ovarian veins 
and para-uterine veins are filled with contrast medium during the arterial phase of post intravenous contrast administration. ${ }^{(2,15,16)}$ Alternatively, this can be identified as OV filling during the nephrographic phase of renal parenchymal enhancement. (6) This imaging modality also assists in grading of ovarian vein reflux as well as the pelvic varicosities detection. ${ }^{(1)}$ In the current study, both approaches were applied for both detection and grading of $\mathrm{OV}$ reflux as well as pelvic varicosities was followed.

Most of the refluxing OVs, $36.76 \%$ were found in patients in their $6^{\text {th }}$ decade. No refluxing veins were detected in the first and second decades. This shows that younger patients are less likely to have reflux while the advancing age coupled with likely increasing parity is associated with venous reflux. Previous study suggested an incidence of multiparous and postmenopausal status in $10 \%$ suffering OV reflux and upto $60 \%$ of them may develop PCS. ${ }^{(1)}$

Normal ovarian veins have valves that maintain antegrade flow of blood from pelvic region to their terminations and towards the heart. Their absence (absent from the orifices of the gonadal veins in $15 \%$ of women) or dysfunction is common and lead to development of primary reflux and pelvic varicosities. ${ }^{(2)}$ In the current study, (17/68) $25 \%$ of the refluxing OVs did not have any associated predisposing factor and were considered to be of primary type.

Pelvic varicosities have also been attributed to mechanical compressive causes, such as abdominal/pelvic masses or uterine malposition causing kinking of the ovarian veins. Obstruction is also attributed to congenital anatomic anomalies such as a retro-aortic left renal vein, compression of the left renal vein by the superior mesenteric artery (nutcracker phenomena), or right $\mathrm{OV}$ compression by common iliac vein. Secondary congestion may be seen with a number of different disorders, including valvular incompetence, portal hypertension, or acquired inferior vena cava syndrome. ${ }^{(17)}$
The current study findings matches these data considering the causes and mechanism of secondary OV reflux among the study group. The bulk, 51/68 (75\%) of refluxing veins in the study, were secondary to abdominal pathologies including abdominal masses, inflammatory, postoperative states, trauma lymphoma, and congenital malformation( four in two patients with absent infra-renal vena cava, one in a patient with double IVC and four in patients who had retroaortic course of left renal vein) and venoocclussive disease. Among these, HCC with cirrhosis with/without portal hypertension (13) and inflammatory disorders (10) formed the largest group of pathologies associated with reflux. However, some pathologies like trauma could not be linked directly with the reflux and all cases would have required review of all previous imaging studies to rule out the likelihood of primary OV reflux that was present before the currently diagnosed reflux in presence acquired pathology. These findings are similar to previously reported findings. ${ }^{(18)}$ Additionally, the study detected the presence of dilated right ovarian vein in a case of IVC occluded by luminal blood thrombus.

Left ovarian veins are commonly affected by reflux due to naturally weak valves ${ }^{(5)}$ and this was also found in the current study where $64.71 \%$ of reflux occurred on the left OV.

The mean age of patients with refluxing OVs was 46.7 years (range 25-75 years). They were mostly in patients in $6^{\text {th }}$ decade $(36.76 \%)$ followed by those in $5^{\text {th }}$ decade $(25 \%)$ and the least in $7^{\text {th }}$ and $8^{\text {th }}$ decades $(5.88 \%)$ with none detected in $1^{\text {st }}$ and $2^{\text {nd }}$ decade. Though direct correlation was not possible, previous studies indicated that this condition is more common in multiparous, premenopausal women who typically present with chronic pelvic pain for more than 6 months with overall population prevalence approaching $\sim 10 \%$ and within this group of patients, up to $60 \%$ may develop pelvic congestion syndrome. ${ }^{(7,8)}$

Tadao $(2004)^{(1)}$, found out that reflux was predominantly seen in multiparous women and 
they suggested that pregnancy has an effect on the development of reflux. In an autopsy study, incompetent ovarian veins were found in multiparous women more often than in uniparous or nulliparous women. The dilation of ovarian veins that normally occurs during pregnancy to compensate for a 60 -fold increase in blood flow is a likely cause of subsequent venous incompetence. $^{(2,9,17,18)}$ This could explain the more incidence noted in above decades in the current study. In addition another study showed that the diameter of the gonadal veins in the younger population ( $\leq 30$ years old) was significantly smaller than that in the elderly population $(\geq 60$ years old) when measured on MDCT and it suggested that it may be related to the parous status of the women. ${ }^{(19)}$

Current study however, contradicted another study which found mean age in the group with dilated and refluxing ovarian veins was 33 years, (20) because the age of their patients was 18-46 years. Calibers of the refluxing veins ranged from $4.1 \mathrm{~mm}$ to $>12 \mathrm{~mm}$ as categorized in the current study. Correlation between the caliber and the reflux rate showed increasing frequency of reflux with the increasing venous caliber.This is comparable to findings by other authors who noted that retrograde flow is typically associated with ovarian vein dilatation. ${ }^{(20)}$

Reflux occurs to variable degrees or extend.In a study by Tadao (2004), ${ }^{(1)}$ the degree of 50 refluxing involving left $\mathrm{OV}$ was analyzed. After assessing the flow-pathway from the level of the kidneys to the pelvic floor, the degree of reflux was divided into three grades. In grade I, retrograde flow remained in the left or right ovarian vein, not reaching the para-uterine veins while in grade II, the retrograde flow extended to the ipsilateral para-uterine veins and not farther. Finally, in grade III, the retrograde flow crossed the midline passing through the uterus from the left into the right para-uterine plexus. They found grade I in four women, grade II in 21 women, and grade III in 25 women.
In the current work, the same grading was applied but also included the right OVs. It found that, refluxing ovarian veins were more (44) on the left side while 24 were on the right side. Grading of refluxing veins showed that most of the refluxing OVs $(38 / 68,55.88 \%)$ were of grade II, followed grade I $(20 / 68,29.41 \%)$ while those of grade III were $10(14.71 \%)$. Overall, grade II reflux was common on both sides, being 10/24 on right and 28/44 on the left side.

These findings are different from those of a previous study which found grade III forming the largest group. ${ }^{(1)}$ This could be explained by inclusion of the right ovarian vein in the current study. However, the increased prevalence in the left side reflux agrees with other studies due to anatomic difference with the left OV having inherently weak or absent venous valves. $(1,13,21)$ Currently, though grade III was the least common, there was statistical significance in its occurrence on the left OVs compared to the right OVs $(\mathrm{p}=0.011)$

\section{Conclusion}

Contrast enhanced MDCT can be used to detect OV reflux and its grade. In addition it helps identify their causes if present. The study shows that secondary OV reflux form a large group and their presence should be searched for to prevent recurrence after treatment. Overall, the left OV was most affected by reflux with grade II OV reflux formed the bulk.

\section{Reference}

1. Hiromura $\mathrm{T}$, Nishioka $\mathrm{T}$, Nishioka $\mathrm{S}$, Ikeda H, Tomita K. Reflux in the left ovarian vein: analysis of MDCT findings in asymptomatic women. American Jou. of Roentgenology 04;183 (5):1411-5.

2. Ignacio EA, Ruchika Dua I, Sarin S, Harper AS, Yim D, Mathur V, et al., editors. Pelvic congestion syndrome: diagnosis and treatment. Seminars in interventional radiology 2008: Thieme Medical Publishers. 
3. Durham JD, Machan L, editors. Pelvic congestion syndrome. Seminars in interventional radiology 2013: Thieme Medical Publishers.

4. Osman MW, Nikolopoulos I, Jayaprakasan $\mathrm{K}$, Raine-Fenning N. Pelvic congestion syndrome. The Obstetrician \& Gynaecologist 2013;15(3):151-7

5. ASCIUTTO G. Pelvic vein incompetence: a review of diagnosis and treatment. History of venous surgery (3) PAGE 59. 2012;19(2):84-90.

6. Kaufman JA, Waltman AC, Rivitz SM, Geller SC. Anatomical observations on the renal veins and inferior vena cava at magnetic resonance angiography. Cardiovascular and interventional radiology 1995;18(3):153-7.

7. Kuligowska E, Deeds III L, Lu III K. Pelvic Pain: Overlooked and Underdiagnosed Gynecologic Conditions. Radiographics 2005;25(1):3-20.

8. Mathias SD, Kuppermann M, Liberman RF, Lipschutz RC, Steege JF. Chronic pelvic pain: prevalence, health-related quality of life, and economic correlates Obstetrics \& Gyn. 1996;87(3):321-7.

9. Beard R, Reginald P, Wadsworth J. Clinical features of women with chronic lower abdominal pain and pelvic congestion. BJOG: An International Journal of Obstetrics \& Gynaecology 1988;95(2):153-61.

10. Cordts PR, Eclavea A, Buckley PJ, DeMaioribus CA, Cockerill ML, Yeager TD. Pelvic congestion syndrome: early clinical results after transcatheter ovarian vein embolization. Journal of vascular surgery 1998;28(5):862-8.

11. Hobbs J. The pelvic congestion syndrome. British journal of hospital medicine 1990;43(3):200-6.

12. Lechter A, Alvarez A, Lopez G. Pelvic varices and gonadal veins. Phlebology. 1987;2(3):181-8.
13. Giacchetto C, Catizone F, Cotroneo G, Cavallaro V, Cammisuli F, Minutolo V, et al. Radiologic anatomy of the genital venous system in female patients with varicocele. Surgery, gynecology \& obstetrics. 1989;169(5):403-7.

14. Coakley FV, Varghese SL, Hricak H. CT and MRI of pelvic varices in women. Journal of computer assisted tomography 1999;23(3):429-34.

15. Desimpelaere J, Seynaeve P, Hagers Y, Appel B, Mortelmans L. Pelvic congestion syndrome: demonstration and diagnosis by helical CT Abdominal imaging 1999;24 (1): 100-2.

16. Eren S. Multi-Detector Row Computed Tomography Findings of Pelvic Congestion Syndrome Caused by Dilated Ovarian Veins The Eurasian Journal of Medicine 2010;42(3):128.

17. Freedman J, Ganeshan A, Crowe P. Pelvic congestion syndrome: the role of interventional radiology in the treatment of chronic pelvic pain. Postgraduate medical journal 2010;86(1022):704-10.

18. Osman MW, Nikolopoulos I, Jayaprakasan K, Raine-Fenning N. Pelvic congestion syndrome. The Obstetrician \& Gynaecologist 2013;15(3):151-7

19. Asayama Y, Yoshimitsu K, Aibe H, Nishie A, Kakihira D, Irie H, et al. MDCT of the gonadal veins in females with large pelvic masses: value in differentiating ovarian versus uterine origin. Ajr 2006;186 (2):440-8.

20. Rozenblit AM, Ricci ZJ, Tuvia J, Amis Jr ES. Incompetent and dilated ovarian veins: a common CT finding in asymptomatic parous women. American Journal of Roentgenology 2001;176(1):119-22.

21. Ahlberg N, Bartley O, Chidekel N. Right and left gonadal veins an anatomical and statistical study. Acta Radiologica (Sweden) 1966;4(6):593-601. 